Article

\title{
Deproteinization as a Rapid Method of Saliva Purification for the Determination of Carbamazepine and Carbamazepine-10,11 Epoxide
}

\author{
Ewelina Dziurkowska *(1) and Marek Wesolowski@ \\ Department of Analytical Chemistry, Medical University of Gdansk, Gen. J. Hallera 107, 80-416 Gdansk, Poland; \\ marek.wesolowski@gumed.edu.pl \\ * Correspondence: elewajs@gumed.edu.pl; Tel.: +48-58-349-1097
}

Received: 26 February 2020; Accepted: 23 March 2020; Published: 27 March 2020

check for updates

\begin{abstract}
Saliva is a valuable diagnostic material that, in some cases, may replace blood. However, because of its different composition, its use requires the development of new, or the modification of existing, extraction procedures. Therefore, the aim of the study was to develop a method of saliva purification that would enable the determination of carbamazepine and its metabolite, carbamazepine-10,11 epoxide. When comparing two methods of sample purification (Solid Phase Extration (SPE) and deproteinization), it was found that the second method yielded more favorable results. A 1\% formic acid solution in acetonitrile was used for extraction. The samples were shaken and centrifuged, and the supernatant obtained was evaporated and dissolved in a mobile phase, then chromatographically analyzed. The developed method was validated by determining its linearity in the range of $10-5000 \mathrm{ng} / \mathrm{mL}$ for both analytes. Intra- and inter-day precision did not exceed $14 \%$. In order to check the usefulness of the method, both analytes were determined in the saliva samples from 20 patients treated with carbamazepine. The content of both analytes was detected and determined in all of the tested samples of saliva. It was found that the method developed is rapid, sensitive, reliable, and can be used to monitor the concentration of carbamazepine and metabolite in patients' saliva.
\end{abstract}

Keywords: saliva; deproteinization; carbamazepine; carbamazepine-10,11 epoxide

\section{Introduction}

For many years, saliva has been the subject of research aimed at making use of it as an easily accessible diagnostic material. Its collection does not require the presence of trained staff, and the patient can take the sample at home and deliver it to the laboratory. Saliva sampling does not cause any additional stress, which is extremely important for determining the levels of substances such as cortisol. Unlike blood sampling, it is also much less stressful to children. Monitoring the level of active substances in saliva can be the method of choice for the elderly, in whom blood collection is hindered by the fragility of veins. Moreover, in the case of persons who have committed a crime, taking a sample of saliva prevents one from falsifying or submitting for analysis a sample received from another person, which may be the case when urine samples are taken [1-3].

Despite its many advantages, for many years, saliva has not been the basic biological material for the analyses of active substances, because very often, the concentration of the monitored substances in saliva is lower than in blood. Therefore, the determination of analytes in saliva requires a more sensitive analytical method or a larger sample volume. Moreover, the composition of saliva is different from that of blood, which necessitates the creation of new methods or the modification of already existing procedures for blood in order to use them for saliva analysis [1,2]. 
When using saliva to monitor the concentration of drugs in the body, special attention should be paid to the correlation between the concentration of the test substance in the blood and saliva. Such a correlation allows for using saliva as a diagnostic material. In the absence of a correlation, saliva may provide a convenient matrix for abuse control, e.g., abuse of psychoactive agents. One of the drugs for which the correlation between differences in the blood and saliva concentration has been confirmed is carbamazepine (Figure 1a) [2-9].

Carbamazepine was discovered in 1953 and introduced into treatments in 1962 as an antiepileptic, as well as being used for treating neuropathic pain. Moreover, in combination with neuroleptics, it is used to treat schizophrenia and as a mood stabilizer in bipolar disorder $[8,10,11]$. The mechanism of carbamazepine is to block the sodium channels. Its half-life after a single administration is $36 \mathrm{~h}$, whereas after repeated administration, due to strong hepatic enzyme induction, the half-life is shortened and ranges between 16 and $24 \mathrm{~h}$. It is metabolized by isoenzyme from the group of cytochrome P450CYP3A4 to active epoxide (carbamazepine-10,11 epoxide; Figure 1b), and is excreted in the urine (72\%) [10-12].

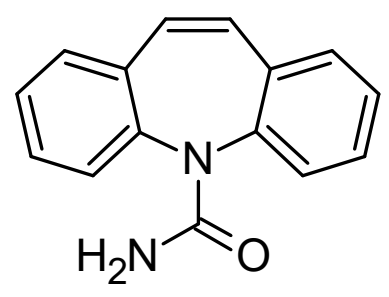

(a)

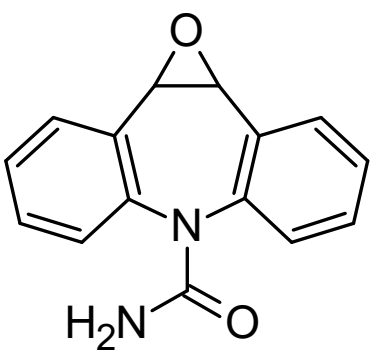

(b)

Figure 1. Chemical formulas of carbamazepine (a) and its active metabolite, carbamazepine-10,11 epoxide (b).

Carbamazepine may affect the metabolism of other drugs metabolized by this isoenzyme, e.g., reducing the concentrations of clozapine, quetiapine, and aripiprazole. When used concurrently with lithium, carbamazepine may intensify lithium's neurotoxic effects. Because of the nonlinear pharmacokinetics of carbamazepine and its active metabolite, as well as the numerous adverse effects and high individual variability in the dose-to-plasma concentration resulting from numerous drug interactions, it is advisable to monitor these analytes and personalize the dose-to-plasma concentration. Carbamazepine and carbamazepine epoxide concentrations in the body should be monitored, particularly in children and adults, when other drugs metabolized by isoenzyme CYP3A4 are administered simultaneously [13]. In this case, monitoring can be carried out using saliva samples.

Saliva sampling for the analysis of the carbamazepine content is most often taken following stimulating its secretion by chewing Parafilm [3,9,14] or eating citric acid [15-18]. Saliva samples can also be collected in a tube without prior stimulation [8-20] or on cotton swabs specially designed for this purpose, which are placed in the mouth to soak up the saliva [21-23]. After removing the swab from the mouth, it is placed in a tube and is centrifuged.

The most common method of carbamazepine isolation from saliva samples is liquid-liquid extraction [8,14,16,24-26]. Saliva samples are also purified using a modified liquid-liquid extraction, cloude-point extraction [26], solid phase extraction [22,23], and saliva deproteinization [15,20].

Carbamazepine in saliva can be quantified by an enzymatic method (FPIA-fluorescence polarization immunoassay) [7,19], as well as by separation techniques, gas chromatography $[2,16,27]$, and liquid chromatography coupled with UV detection $[3,8,9,14,15,17,18,20-26]$.

Taking into account the potential benefits that may arise from the use of saliva as a diagnostic material, the aim of this study was to develop extraction conditions and to validate a method for the determination of carbamazepine and its active metabolite in saliva samples. The intention was to come up with an easy, rapid, and accurate method of saliva sample purification, and the volume 
of sample needed to determine the concentration of analytes. The developed method requires only $200 \mu \mathrm{L}$ of saliva, which is significant, as the determinations using saliva are particularly recommended for children from whom blood collection may be very stressful.

The significance and newness in the developed method in relation to the existing ones is based on the fact that the developed method allows for the determination of carbamazepine and its metabolite in a small saliva sample collected with salivette, which is used to help with secretion of an appropriate amount of saliva. Moreover, our study revealed that the Solide Phase Extraction (SPE) Phree columns designed for analysing small blood volumes cannot be used for saliva purification. However, the use of deproteinization was the most effective for this purpose.

\section{Experimental Section}

\subsection{Instruments}

The chromatographic analysis was performed with a Nexer XR UHPLC (Ultra High Performance Liquid Chromatography) liquid chromatograph (Schimadzu, Kyoto, Japan), equipped with a SPD-M30A UV-Vis detector with a diode array and a highly sensitive measuring cell SPD-M30A (85 mm), CBM-20 Alite control system, LC-30AD pump, SIL-30AC autosampler, and CTO-20AC thermostat (Shimadzu, Kyoto, Japan). The compounds were separated using a Luna Omega $1.6 \mu \mathrm{m}$ Polar C18 (LC Column $50 \times 2.1 \mathrm{~mm}$ ID) with pre-columns (UHPLC Fully Porous Polar C18, $2.1 \mathrm{~mm}$ ID). The compounds were eluted from the column at $35{ }^{\circ} \mathrm{C}$ with a mobile phase consisting of water with added formic acid and triethylamine (TEA) (solvent A, pH 4.2) and acetonitrile (solvent B) at a flow of $1 \mathrm{~mL} / \mathrm{min}$. The composition of the mobile phase and its changes during the analysis are presented in Table 1.

Table 1. UHPLC (Ultra High Performance Liquid Chromatography) gradient program.

\begin{tabular}{cc}
\hline Time $(\mathbf{m i n})$ & Gradient (Percentage of Solvent B by Volume) \\
\hline 0.01 & 10 \\
0.5 & 10 \\
10.0 & 55 \\
12.0 & 90 \\
12.2 & 10 \\
15.0 & 10 \\
\hline
\end{tabular}

\subsection{Chemicals and Solvents}

Acetonitrile, methanol, and formic acid were obtained from POCh (Gliwice, Poland), and TEA was supplied by Sigma-Aldrich (St. Louis, MO, USA). All of the reagents had HPLC super grade purity. The deionized water was purified by Ultra-Toc/UV, Hydrolab (Straszyn, Poland). Standards of carbamazepine came from Polpharma (Starogard Gdański, Poland). Carbamazepine-10,11 epoxide solution (1 mg/mL) was obtained from Sigma-Aldrich (St. Louis, MO, USA). The internal standard (IS), chlordiazepoxide, was purchased from Polfa Tarchomin (Warsaw, Poland).

Standard solutions of carbamazepine and chlordiazepoxide $(1 \mathrm{mg} / \mathrm{mL})$ were prepared by weighing $10 \mathrm{mg}$ of the substance and dissolving in $10 \mathrm{~mL}$ of methanol. Working solutions of carbamazepine, carbamazepine-10,11 epoxide, and chlordiazepoxide were prepared by diluting the standard solutions with methanol. All of the working solutions were stored at $-21^{\circ} \mathrm{C}$.

\subsection{Saliva Sampling}

Saliva samples for preliminary examination and method validation were taken using Salivettes (Sarstedt, Nümbrecht, Germany). Healthy volunteers were required to refrain from eating and drinking liquids $30 \mathrm{~min}$ before sampling, and were asked to wash their mouth with water $10 \mathrm{~min}$ before placing a Salivette in their mouths and chewing it for about $2 \mathrm{~min}$. The swab was then placed back in the 
tube and centrifuged for $5 \mathrm{~min}$ at $8000 \mathrm{rpm}$. Centrifuged samples were frozen and stored at $-20^{\circ} \mathrm{C}$ until analysis.

\subsection{Saliva Treatment}

The saliva samples were thawed, centrifuged, and then $200 \mu \mathrm{L}$ of test material was collected and placed in plastic tubes. A $1 \%$ formic acid solution in acetonitrile $(600 \mu \mathrm{L})$ was added to each of them, mixed with a vortex, shaken (laboratory shaker 358 S, Elpin, Poland) for about 10 min, and centrifuged for $5 \mathrm{~min}$ at $8000 \mathrm{rpm}$. The supernatant was transferred to glass tubes and evaporated. The dry residue was dissolved in $100 \mu \mathrm{L}$ of an acetonitrile/water mixture with formic acid (1:10), and $10 \mu \mathrm{L}$ was injected into the chromatographic column.

\subsection{Method Validation}

\subsubsection{Linearity}

The linearity of the method was determined by performing four calibration curves on four consecutive days. The curves were prepared by adding appropriate volumes of working solutions to $200 \mu \mathrm{L}$ of saliva, so that the final IS concentration was $500 \mathrm{ng} / \mathrm{mL}$, while the analytes were 10,50 , $100,500,1000,3000$, and $5000 \mathrm{ng} / \mathrm{mL}$, respectively. The acceptance criteria for the calibration curve included a coefficient of determination (R2) $\geq 0.99$ and residuals $\leq 15 \%$ at each concentration level.

\subsubsection{Selectivity}

The selectivity of the method was determined by analyzing 10 blank saliva samples obtained from 10 healthy volunteers. The study was conducted to detect interference resulting from the presence of endogenous compounds. Their absence allows for the method to be considered selective.

\subsubsection{Precision and Accuracy}

Intra- and inter-day precision and accuracy were tested at three concentrations ( $30 \mathrm{ng} / \mathrm{mL}-\mathrm{low}$ quality control (QC); $2000 \mathrm{ng} / \mathrm{mL}$-medium QC; $3750 \mathrm{ng} / \mathrm{mL}$ - high QC). For intra-day precision, five samples of each concentration were analyzed on one day. In the case of inter-day precision, one series of each concentration was analyzed over four consecutive days $(n=20)$. The precision of the method was expressed by the coefficient of variation (\%CV), taking $\leq 15 \%$ as an acceptable value. The accuracy was expressed as the percentage of nominal concentration, with the threshold within $85-115 \%$ of the target concentration.

\subsubsection{Limits of Quantification}

The limit of quantification (LOQ) for the tested concentrations was repeated five times, and its value was considered to be the lowest concentration of analytes for which the signal was ten times higher than the noise, the precision of CV determination was $<20 \%$, while the recovery of the analyte was $\pm 20 \%$.

\subsubsection{Absolute Recovery and Extraction Recovery}

The absolute recovery and extraction recovery were determined for two concentrations of analytes (30 ng/mL-QC low; $3750 \mathrm{ng} / \mathrm{mL}$-high QC). Six saliva samples were prepared for each concentration by adding to each sample an appropriate volume of working solution of the analyte and IS. The samples were analyzed according to the procedures described in Sections 2.1 and 2.4.

In order to determine the absolute recovery, the peak areas of the extracted analytes were compared with the peak areas obtained from the analysis of six neat standards of each concentration. The extraction recovery was determined by comparing the surface areas of the peaks of the analytes subjected to the extraction process, with the peak areas of each concentration of the analytes obtained by analyzing blank saliva samples, which were loaded with the solutions of the analytes after extraction. 
The average of six neat standards of each concentration was assumed to be $100 \%$, while an acceptable result was considered to be the one for which the analyte's value exceeded $50 \%$.

\subsubsection{Stability}

The stability of the analytes was tested for three concentrations (low QC, medium QC, and high QC). The stability of the analytes was determined both in the matrix during storage at $8{ }^{\circ} \mathrm{C}$ and in a freeze-thaw test, during which spiked saliva samples were stored at $-21^{\circ} \mathrm{C}$. For each concentration of carbamazepine and metabolite, three $800 \mu \mathrm{L}$ saliva samples were prepared in plastic tubes, spiked with an appropriate volume of analytes, and mixed. Then, $200 \mu \mathrm{L}$ of saliva was taken from each sample, IS added, extracted, and chromatographed according to the procedures described in Sections 2.4 and 2.5. The remainder of the sample was placed back in the refrigerator or frozen and analyzed in the following days.

The stability of the analytes was also determined during their storage in an autosampler at $15^{\circ} \mathrm{C}$ for $72 \mathrm{~h}$. For this purpose, five samples of each concentration were extracted and chromatographed. After $72 \mathrm{~h}$, the samples were then chromatographed again alongside a freshly prepared calibration curve.

The compounds whose concentration decreased by less than $15 \%$ under the given storage conditions were considered to be stable.

\subsection{Clinical Application}

The usefulness of the method was determined by examining the saliva samples of 20 patients treated with carbamazepine preparations. They were collected from patients of the Nervous and Mentally Ill Hospital in Starogard Gdański (Poland). The study protocol was approved by the ethical committee of the Medical University of Gdansk, Poland (NKBBN/139/2016). Saliva was taken using Salivettes about $2 \mathrm{~h}$ after the administration of carbamazepine. The Salivette was placed in a test tube and centrifuged, and the obtained filtrate was frozen at $-21^{\circ} \mathrm{C}$ and stored until analysis. To perform the analyses, the samples were thawed, centrifuged, and $200 \mu \mathrm{L}$ of liquid taken. Then, $50 \mu \mathrm{L}$ of IS solution $(10 \mu \mathrm{g} / \mathrm{mL})$ was added and the procedure described in Section 2.4 was followed.

\section{Results}

\subsection{Chromatographic Analysis}

The first stage of chromatographic separation optimization included the selection of the composition of the mobile phase, its flow rate, and the internal standard. The composition of the mobile phase and its appropriate flow ensured the optimal separation of the analyzed substances from other components of the matrix. Chromatographic analysis lasted $15 \mathrm{~min}$ and peak detection was performed at $240 \mathrm{~nm}$. Chlordiazepoxide was used as the IS. This benzodiazepine derivative is not currently used in medicine, and therefore may not be present in patients' saliva. Furthermore, chlordiazepoxide shows good UV absorption at $240 \mathrm{~nm}$. The chromatogram obtained by analyzing standard solutions using optimized chromatographic analysis conditions is shown in Figure 2.

\subsection{Method Development}

\subsubsection{Extraction with Phree Columns}

Phree phospholipid removal plates (Phenomenex, Torrance, CA, USA) were used to optimize the purification of the saliva samples for the small sample volumes. Carbamazepine is well absorbed from the digestive tract, and usually occurs in high concentrations in saliva. This allows for the use of a small sample volume ( $200 \mu \mathrm{L})$. Preliminary studies showed that the use of Strata X-C columns, which were rinsed with water and a mixture of water and methanol (1:1), and saliva samples diluted with $2 \%$ formic acid solution and a mixture of water and methanol (1:1), is a good procedure for purifying saliva samples [22]. Applied to the analysis of neuroleptics and carbamazepine, it allowed for good 
saliva purification and an analysis of patient samples. However, when the saliva contained the active metabolite carbamazepine, the extraction process was inefficient, as evidenced by the relatively low recovery [23]. Therefore, it was decided to use Phree columns, which do not require activation, and the purification process is simplified by adding to the analyzed sample a $1 \%$ formic acid solution in methanol or acetonitrile as a deproteinizing agent. If a methanol solution is used, the ratio of sample volume to solution is 1:4, and if acetonitrile is used, the ratio is 1:3. In both cases, after the addition of the deproteinizing solution, the samples were shaken for $10 \mathrm{~min}$, centrifuged, and then applied to the columns. The obtained extract was evaporated and the dry residue was dissolved in the mobile phase. However, the chromatographic analysis of blank saliva samples showed that in both cases, there was no complete purification of the extracts, and the retention times of the recorded peaks coincided with those of the analytes. This makes it impossible to analyze low concentrations of carbamazepine and its metabolite in saliva, as illustrated in Figure 3a,b.

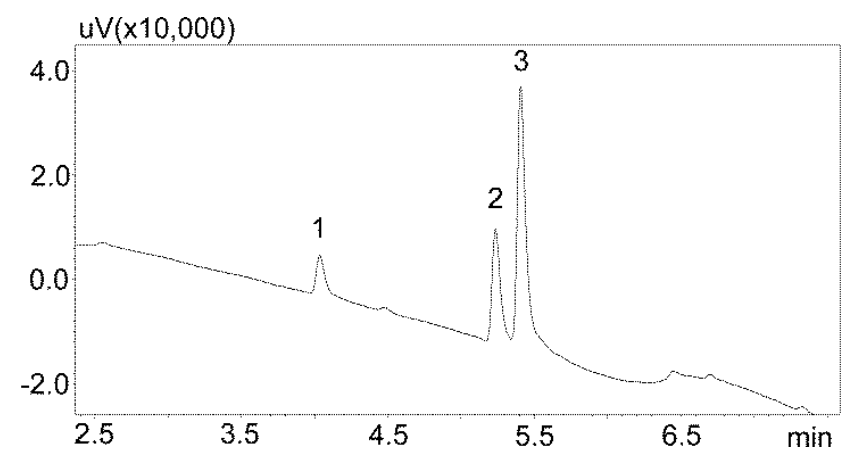

Figure 2. Chromatogram of the standard solutions obtained by optimized UHPLC. The compounds were separated using a Luna Omega $1.6 \mu \mathrm{m}$ Polar C18 column and a mobile phase consisting of water with formic acid and (solvent A) and acetonitrile (solvent B) at a flow of $1 \mathrm{~mL} / \mathrm{min}$. 1-carbamzepine-10,11 epoxide; 2-carbamazepine; 3-IS (internal standard: chlordiazepoxide).
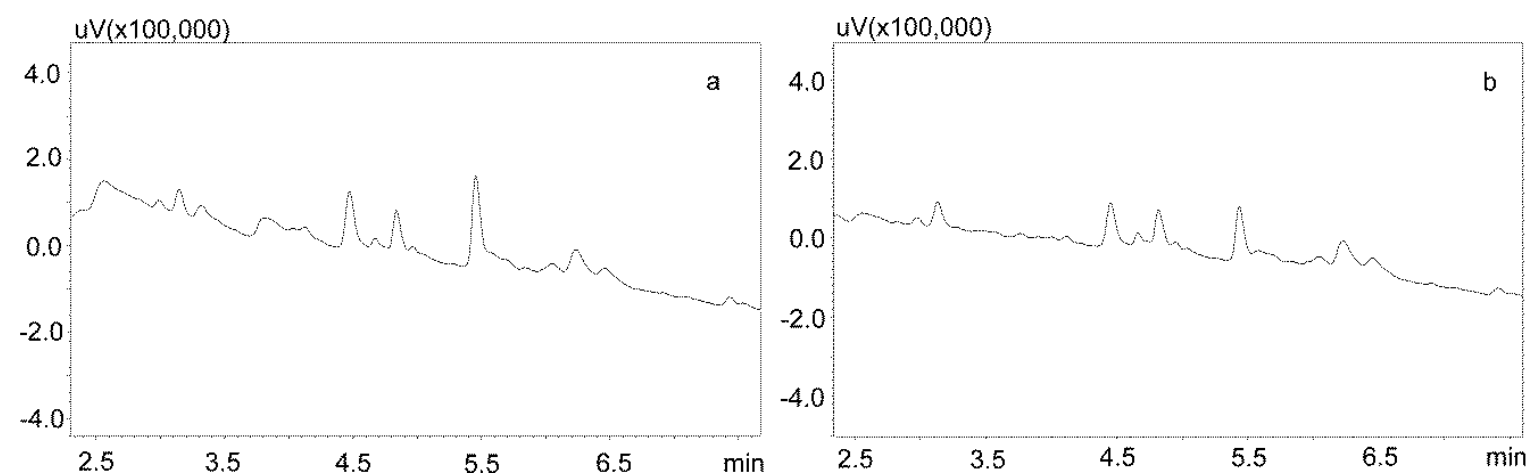

Figure 3. Chromatograms of blank saliva extracted with Phree columns: (a) extracted with $1 \%$ formic acid in methanol; (b) extracted with $1 \%$ formic acid in acetonitrile. The separation was carried out using a Luna Omega $1.6 \mu \mathrm{m}$ Polar C18 column and a mobile phase consisting of water with formic acid (solvent A) and acetonitrile (solvent B) at a flow of $1 \mathrm{~mL} / \mathrm{min}$.

\subsubsection{Modification of the Deproteinization Process}

For the modification of the sample purification, deproteinization was applied using the same formic acid solutions as for the purification of saliva using the Phree columns (Section 3.2.1.). Then, 800 or $600 \mu \mathrm{L}$ of $1 \%$ formic acid solution in methanol or acetonitrile were added to $200 \mu \mathrm{L}$ of saliva, respectively, mixed, shaken for $10 \mathrm{~min}$, centrifuged, and then $10 \mu \mathrm{L}$ of supernatant was analyzed by UHPLC. This procedure allowed for a very good purification of blank saliva samples, as illustrated by Figure $4 a, b$. However, in the case of spiked samples, regardless of the deproteinization solution used, 
the shape of the peaks was unsatisfactory, as they were very wide at the base. This caused difficulties in determining their surface area for low concentrations of the analyzed compounds (Figure 5a).
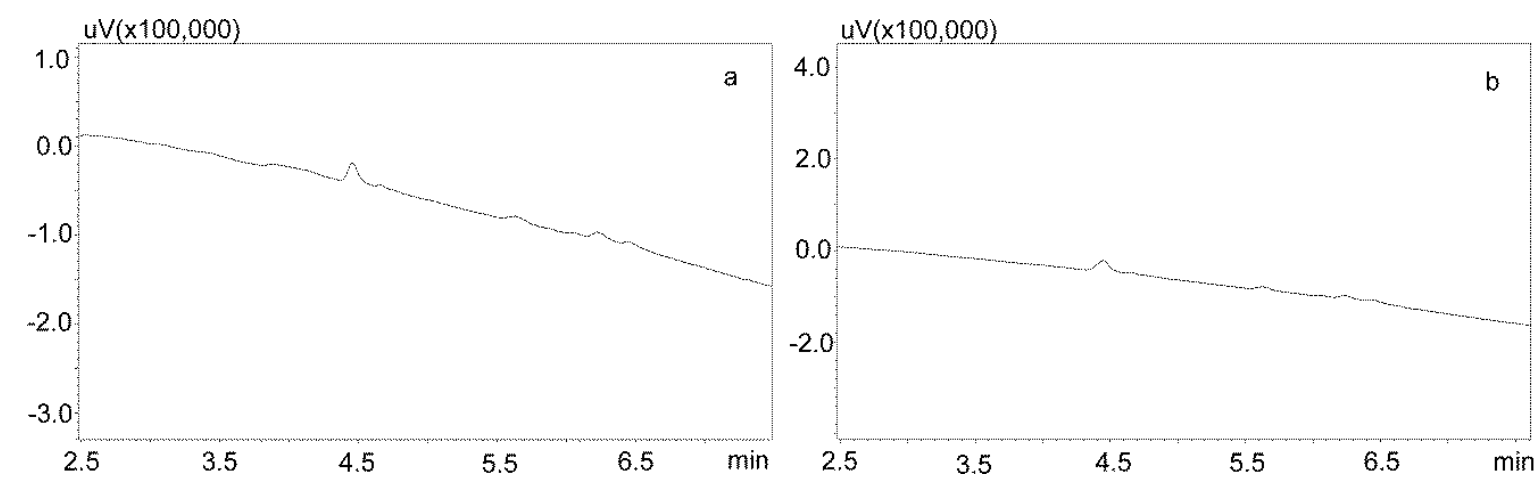

Figure 4. Chromatograms of blank saliva samples deproteinized with: (a) 1\% formic acid in methanol; (b) $1 \%$ formic acid in acetonitrile. The separation was carried out using a Luna Omega $1.6 \mu \mathrm{m}$ Polar C18 column and a mobile phase consisting of water with formic acid (solvent A) and acetonitrile (solvent B) at a flow of $1 \mathrm{~mL} / \mathrm{min}$.

Another modification of the purification of saliva containing carbamazepine and its active metabolite included the evaporation of a formic acid solution and the dissolution of the dry residue in the mobile phase. In addition, to reduce the use of solvents, and because of the small differences in the amount of impurities visible on the chromatograms after deproteinization, a $1 \%$ formic acid solution in acetonitrile was used. For this purpose, the supernatant was evaporated and the dry residue after dissolution in the mobile phase was centrifuged again and analyzed chromatographically (Figure 5b).
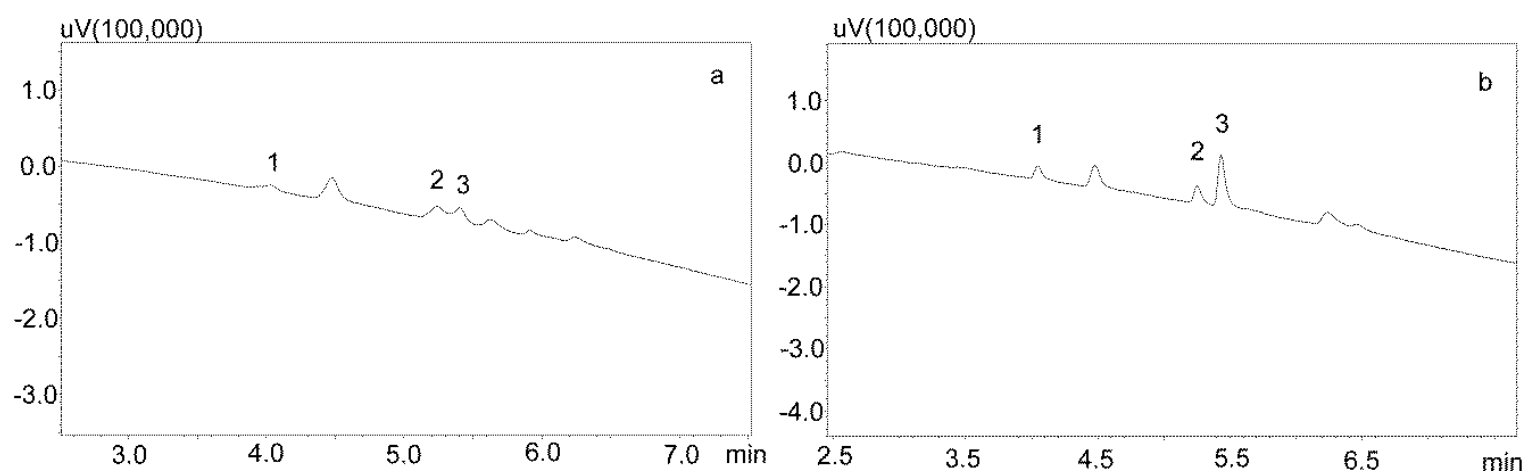

Figure 5. Chromatograms of saliva sample spiked with $1000 \mathrm{ng} / \mathrm{mL}$ after deproteinization with $1 \%$ formic acid in acetonitrile. The compounds were separated using a Luna Omega 1.6 $\mu \mathrm{m}$ Polar C18 column and a mobile phase consisting of water with formic acid (solvent A) and acetonitrile (solvent B) at a flow of $1 \mathrm{~mL} / \mathrm{min}$. (a) without evaporation; (b) after evaporation and dissolving in mobile phase. 1-carbamzepine-10,11 epoxide; 2-carbamazepine; 3-IS (chlordiazepoxide).

\subsection{Method validation}

The linearity specified for both analytes in the concentration range $10-5000 \mathrm{ng} / \mathrm{mL}$ has shown that the method is linear and meets the specified acceptance criterion $\left(R^{2} \geq 0.99\right.$, Table 2$)$. The LOQ for both analytes was set at $10 \mathrm{ng} / \mathrm{mL}$. The detailed test results in Table 3 also indicate that the developed method is precise. CV for inter- and intra-day precision did not exceed $15 \%$ for any of the tested concentrations. 
Table 2. Calibration curves and validation parameters for the developed procedure. SD—standard deviation; LOQ-limit of quantification.

\begin{tabular}{ccc}
\hline Calibration curve $y=a x+b(n=4)$ & Carbamazepine & Carbamazepine-10,11 epoxide \\
\hline Range $(\mathrm{ng} / \mathrm{mL})$ & $10-5000$ & $10-5000$ \\
Determination Coefficient $\left(\mathrm{R}^{2}\right)$ & $0.99958 \pm 0.000171$ & $0.99975 \pm 0.000006$ \\
Slope $\mathrm{a} \pm \mathrm{SD}$ & $0.00025 \pm 0.0001$ & $0.000175 \pm 0.00015$ \\
Intercept $\mathrm{b} \pm \mathrm{SD}$ & $0.004825 \pm 0.002326$ & $0.004875 \pm 0.003333$ \\
LOQ $(\mathrm{ng} / \mathrm{mL})$ & 10 & 10 \\
\hline a-slope; b-intercept; SD—standard deviation; LOQ—limit of quantification.
\end{tabular}

Table 3. Intra- and inter-day validation parameters $(n=20)$. CV-coefficient of variation.

\begin{tabular}{ccccccc}
\hline Analyte & \multicolumn{2}{c}{ Carbamazepine } & \multicolumn{3}{c}{ Carbamazepine-10,11 epoxide } \\
\hline Quality Concentration & $\begin{array}{c}\text { Intra-day } \\
(\% \mathrm{CV})\end{array}$ & $\begin{array}{c}\text { Inter-day } \\
(\% \mathrm{CV})\end{array}$ & $\begin{array}{c}\text { Accuracy } \\
(\%)\end{array}$ & $\begin{array}{c}\text { Intra-day } \\
(\% \mathrm{CV})\end{array}$ & $\begin{array}{c}\text { Inter-day } \\
(\% \mathrm{CV})\end{array}$ & $\begin{array}{c}\text { Accuracy } \\
(\%)\end{array}$ \\
\hline Low $(30 \mathrm{ng} / \mathrm{mL})$ & 6.39 & 12.82 & 95.35 & 6.02 & 13.58 & 109.28 \\
Medium $(2000 \mathrm{ng} / \mathrm{mL})$ & 4.97 & 5.32 & 98.51 & 4.18 & 3.14 & 97.59 \\
High $(3750 \mathrm{ng} / \mathrm{mL})$ & 2.69 & 2.67 & 98.65 & 2.67 & 2.77 & 97.93 \\
\hline
\end{tabular}

CV—coefficient of variation.

The selectivity of the method was determined by analyzing 10 blank saliva samples obtained from 10 healthy volunteers. No interference resulting from the presence of endogenous compounds was observed in any of the tested samples, which confirms the absence of peaks with retention times similar to those of analytes.

\subsection{Extraction and Absolute Recovery}

The extraction recovery was determined for two concentrations of analytes $(30 \mathrm{ng} / \mathrm{mL}-$ low $\mathrm{QC}$; $3750 \mathrm{ng} / \mathrm{mL}$ - high QC), comparing the peak areas of the analytes extracted by the method developed with those of blank saliva samples loaded after deproteinization and solution evaporation. For both analytes, extraction recovery was close to $100 \%$. A slightly lower value ( $>95 \%)$ was obtained for the saliva loaded with carbamazepine epoxide at $30 \mathrm{ng} / \mathrm{mL}$.

The absolute recovery was also determined for two concentrations of analytes ( $30 \mathrm{ng} / \mathrm{mL}-\mathrm{low}$ QC; $3750 \mathrm{ng} / \mathrm{mL}$ - high QC), comparing the peak areas of the extracted analytes with the peak areas obtained during the analysis of six neat standards at each concentration. The lowest absolute recovery value ( $>81 \%$ ) was observed for $30 \mathrm{ng} / \mathrm{mL}$, for carbamazepine and its active metabolite.

Both the extraction recovery and absolute recovery for both analytes at all of the tested concentrations met the criteria $(>50 \%)$. Therefore, it should be considered that the method developed for saliva purification is suitable for the determination of carbamazepine and carbamazepine epoxide, as presented in Table 4.

Table 4. Extraction and absolute recovery of carbamazepine and its active metabolite.

\begin{tabular}{ccccc}
\hline Analyte & \multicolumn{2}{c}{ Carbamazepine } & \multicolumn{2}{c}{ Carbamazepine-10,11 epoxide } \\
\hline Concentration (ng/mL) & 30 & 3750 & 30 & 3750 \\
Extraction Recovery (\%) & 99.09 & 101.19 & 95.89 & 97.37 \\
Absolute Recovery (\%) & 82.19 & 97.07 & 81.13 & 92.61 \\
\hline
\end{tabular}

\subsection{Stability}

The stability of analytes in the biological matrix and during the storage of samples in the autosampler was tested. For three concentrations (30 ng/mL-low QC; $2000 \mathrm{ng} / \mathrm{mL}$-medium QC; $3750 \mathrm{ng} / \mathrm{mL}$ - high QC), the results obtained in Table 5 indicate that carbamazepine and carbamazepine-10,11 epoxide are stable under the tested conditions. 
Table 5. Stability study of analytes in spiked saliva stored in a fridge at $8{ }^{\circ} \mathrm{C}$ and after freeze-thaw cycles at $-21{ }^{\circ} \mathrm{C}$. The stability of the extracts kept in the autosampler at $15^{\circ} \mathrm{C}$ for $72 \mathrm{~h}$. Values in the table are expressed as \%loss.

\begin{tabular}{ccccccccccc}
\hline \multirow{2}{*}{ Analyte } & \multicolumn{3}{c}{$-\mathbf{2 1}{ }^{\circ} \mathbf{C}$} & \multicolumn{3}{c}{$\mathbf{8}^{\circ} \mathbf{C}$} & \multicolumn{3}{c}{$\mathbf{1 5}{ }^{\circ} \mathbf{C}$} \\
\cline { 2 - 10 } & $\mathbf{3 0}$ & $\mathbf{2 0 0 0}$ & $\mathbf{3 7 5 0}$ & $\mathbf{3 0}$ & $\mathbf{2 0 0 0}$ & $\mathbf{3 7 5 0}$ & $\mathbf{3 0}$ & $\mathbf{2 0 0 0}$ & $\mathbf{3 7 5 0}$ \\
\cline { 2 - 10 } & & \multicolumn{8}{c}{ Difference (\%) } \\
\hline Carbamazepine & -2.25 & -2.16 & -1.44 & -3.91 & -2.53 & -1.93 & -0.30 & -1.12 & -1.72 \\
\hline Carbamazepine-10,11 epoxide & -2.45 & -1.92 & -0.48 & -6.23 & -3.56 & -2.75 & -3.64 & -2.3 & 0.38 \\
\hline
\end{tabular}

\subsection{Clinical Application}

The saliva samples came from 20 patients treated with carbamazepine in mono- and poly-therapy. The method used allowed for determining the concentrations of both analytes in all of the samples. Detailed data, including age, gender, drug dose, and concentration of analytes in saliva, are presented in Table 6, while examples of patients' saliva extract chromatograms are shown in Figure 6. The average age of the patients was 40 years. The mean concentration of carbamazepine determined in the male saliva was $1882 \mathrm{ng} / \mathrm{mL}$, and was more than twice as high as in the female saliva $(967 \mathrm{ng} / \mathrm{mL})$. The mean metabolite concentration was similar, and was $299 \mathrm{ng} / \mathrm{mL}$ for men and and $249 \mathrm{ng} / \mathrm{mL}$ for women.

Table 6. Patients data. Concentrations of carbamazepine and carbamazepine-10,11 epoxide found in the saliva of 20 patients male $(\mathrm{M})$ and female $(\mathrm{F})$ patients treated with carbamazeine.

\begin{tabular}{cccccc}
\hline Patient & Gender & Age (Year) & Dose $(\mathbf{m g}$ /day) & $\begin{array}{c}\text { Carbamazepine } \\
\text { Concentration } \\
(\mathbf{n g} / \mathbf{m L})\end{array}$ & $\begin{array}{c}\text { Carbamazepine-10,11 } \\
\text { epoxide Concentration } \\
\text { (ng/mL) }\end{array}$ \\
\hline 1 & M & 31 & 800 & 1192.3 & 231.8 \\
2 & M & 44 & 400 & 1817.8 & 428.0 \\
3 & M & 50 & 400 & 4891.0 & 226.8 \\
4 & M & 58 & 800 & 4839.8 & 348.5 \\
5 & M & 49 & 700 & 1165.7 & 229.8 \\
6 & M & 33 & 400 & 262.2 & 120.7 \\
7 & M & 46 & 800 & 2334.2 & 664.1 \\
8 & M & 44 & 800 & 344.7 & 122.0 \\
9 & M & 55 & 400 & 690.7 & 168.9 \\
10 & M & 51 & 800 & 1285.1 & 448.3 \\
11 & F & 22 & 1200 & 464.0 & 161.1 \\
12 & F & 49 & 400 & 282.2 & 127.5 \\
13 & F & 26 & 600 & 472.9 & 172.9 \\
14 & F & 37 & 1200 & 2456.8 & 559.1 \\
15 & F & 31 & 1200 & 1668.1 & 569.1 \\
16 & F & 29 & 1800 & 613.5 & 182.1 \\
17 & F & 19 & 600 & 784.3 & 273.3 \\
18 & F & 31 & 800 & 1119.9 & 152.7 \\
19 & F & 69 & 900 & 607.6 & 147.7 \\
20 & F & 30 & 400 & 1198.2 & 142.7 \\
\hline
\end{tabular}



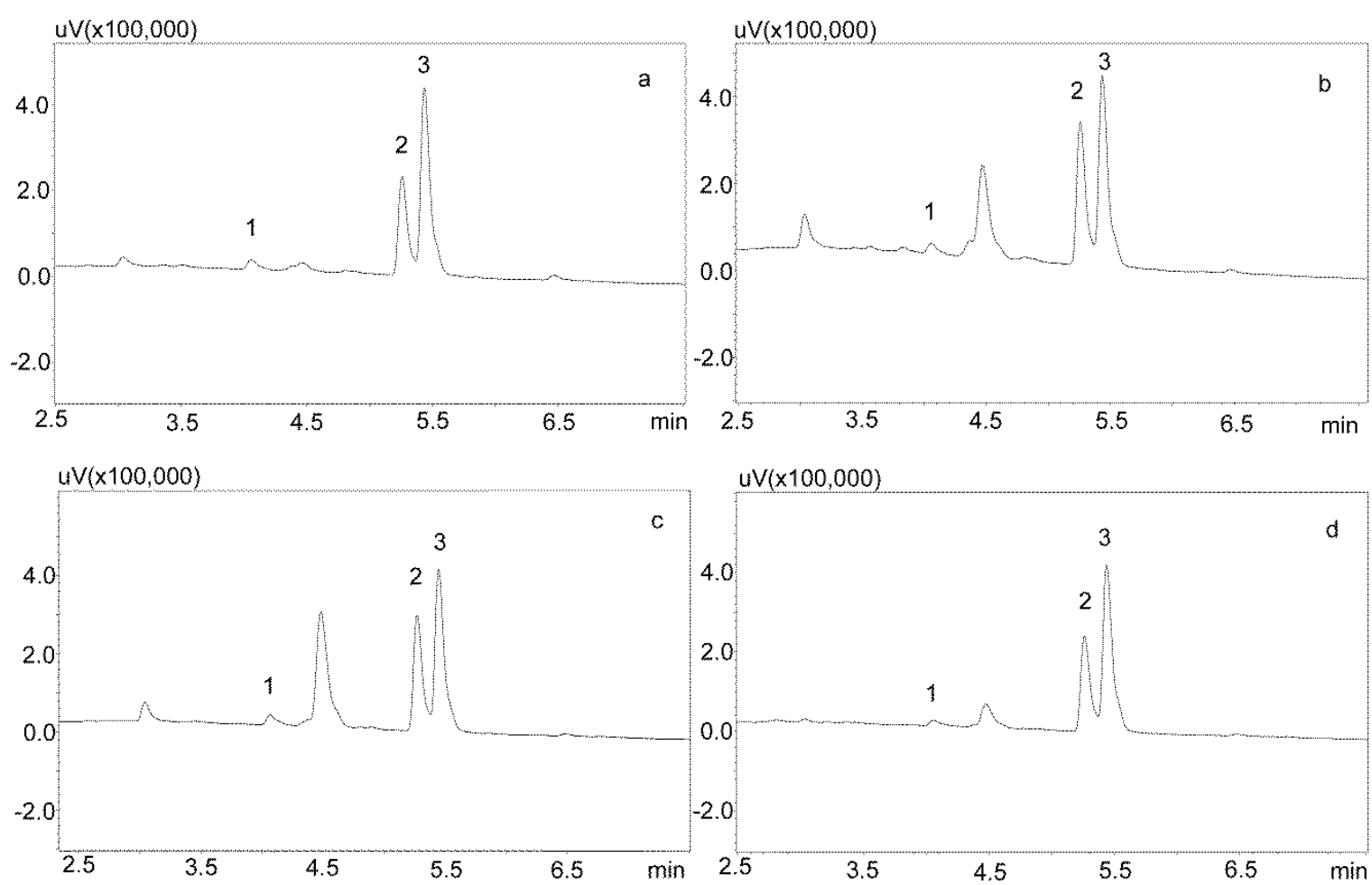

Figure 6. Chromatogram of saliva extracts of patients treated with carbamazepine: 1 -carbamzepine-10,11 epoxide; 2-carbamazepine; 3-IS (chlordiazepoxide). (a) Patient 15: $569.1 \mathrm{ng} / \mathrm{mL}$ (1), $1668.1 \mathrm{ng} / \mathrm{mL}$ (2); (b) Patient 14: 559.1 ng/mL (1), 2456.8 ng/mL (2); (c) Patient 7: 664.1 ng/mL (1), 2334.2 ng/mL (2); (d) Patient 2: $428 \mathrm{ng} / \mathrm{mL}(1), 1817.8 \mathrm{ng} / \mathrm{mL}$ (2). The compounds were separated using a Luna Omega $1.6 \mu \mathrm{m}$ Polar C18 column and a mobile phase consisting of water with formic acid (solvent A) and acetonitrile (solvent $\mathrm{B}$ ) at a flow of $1 \mathrm{~mL} / \mathrm{min}$.

\section{Discussion}

The method developed in this study enables the determination of carbamazepine and carbamazepine-10,11 epoxide in human saliva. Although carbamazepine was introduced to the treatment as early as the 1960s, it is still one of the most frequently used substances in central nervous system diseases. It is also used in the pharmacotherapy of epilepsy in children. Carbamazepine has a strong influence on its own metabolism and the drugs used in parallel, and so it is advisable to monitor its concentration in the body.

As a result of the confirmed correlation of its concentrations in blood and saliva, various studies have been carried out to develop methods of carbamazepine determination in these diagnostic materials. The methods for the determination of anti-epileptic drugs in blood have often been modified to determine carbamazepine in saliva $[2,3,6,8,9,17,18]$. Most often, saliva samples were $1 \mathrm{~mL}[14,18,22,23,25]$ or more $[3,6,8]$, and were rarely less than $1 \mathrm{~mL}[15,21,24,27]$. The method developed in this study allows for the determination of carbamazepine and its active metabolite, in as little as $200 \mu \mathrm{L}$ of saliva. This is particularly useful in children and those carbamazepine users who suffer from dry mouth.

The developed method is characterized by a good linearity in the concentration range of $10-5000 \mathrm{ng} / \mathrm{mL}(\mathrm{R} 2>0.999)$. The concentration range was selected to coincide with the range of therapeutic concentrations observed in patients' saliva $[4,6-8,15,16]$.

Purification of saliva samples involved deproteinization, which proved to be the simplest and most effective method of extraction of the analyzed analytes. In many studies, saliva is purified by liquid-liquid extraction $[8,14,16,24-26]$, which provides a high recovery of analytes $>89 \%$ [24,25], but requires volatile and toxic solvents such as chloroform. In turn, the use of SPE does not need toxic 
solvents, but carbamazepine interacts strongly with the adsorbent, which reduces its recovery [23]. In this study, the extraction recovery of the developed method exceeded $95 \%$ and the absolute recovery exceeded $81 \%$ for both analytes.

All previous literature reports confirm that the analyzed compounds are persistent and their decomposition during storage does not exceed 10\% [20]. These studies also confirm the stability of the analytes under the tested conditions. The highest concentration reduction, at $6 \%$, was recorded for $30 \mathrm{ng} / \mathrm{mL}$ carbamazepine epoxide in saliva samples stored at $8^{\circ} \mathrm{C}$.

The developed method allows for the determination of concentrations of both analytes in all of the tested patient samples. The determined concentrations ranged from 262 to $4891 \mathrm{ng} / \mathrm{mL}$ for carbamazepine, and from 120 to $664 \mathrm{ng} / \mathrm{mL}$ carbamazepine-10,11 epoxide, which is consistent with the literature data $[4,6,8]$.

\section{Conclusions}

The aim of this study was to develop a method for saliva purification and to determine the concentration of carbamazepine and its active metabolite, carbamazepine-10,11 epoxide. Among the extraction methods studied, deproteinization with $1 \%$ formic acid solution in acetonitrile proved to be the most effective. The volume of the sample used for analysis does not exceed $200 \mu \mathrm{L}$. The method is linear in the range $10-5000 \mathrm{ng} / \mathrm{mL}\left(R^{2}>0.999\right)$, and is characterized by high precision, with the highest CV of $12.82 \%$ for carbamazepine and $13.58 \%$ for carbamazepine epoxide. Moreover, the extraction efficiency exceeded $95 \%$, and the absolute recovery exceeded $81 \%$. Both carbamazepine and its active metabolite were stable under all of the tested conditions. Using the developed method, the concentrations of both analyzed compounds were determined in all of the saliva samples from the patients.

Author Contributions: Conceptualization, E.D.; methodology, E.D.; validation, E.D.; formal analysis, E.D.; investigation, E.D.; writing (original draft preparation), E.D.; writing (review and editing), M.W.; supervision, M.W.; funding acquisition, E.D. and M.W. All authors have read and agreed to the published version of the manuscript.

Funding: This research was funded by the National Science Centre (NCN), Poland, under the Miniatura 1 grant, number 2017/01/X/NZ7/01080, and by the Ministry of Science and Higher Education, Poland, grant number 02-0015/07/505.

Conflicts of Interest: The authors declare no conflict of interest.

\section{References}

1. Caporossi, L.; Santoro, A.; Papaleo, B. Saliva as an analytical matrix: State of the art and application for biomonitoring. Biomarkers 2010, 15, 475-487. [CrossRef]

2. Rylance, G.W.; Moreland, T.A. Saliva carbamazepine and phenytoin level monitoring. Arch. Dis. Child. 1981, 56, 637-652. [CrossRef]

3. Eeg-Olofsson, O.; Nilsson, H.L.; Tonnby, B.; Arvidsson, J.; Grahn, P.A.; Gylje, H.; Larsson, C.; Norén, L. Diurnal variation of carbamazepine and carbamazepine-10,11-epoxide in plasma and saliva in children with epilepsy: A comparison between conventional and slow-release formulations. J. Child. Neurol. 1990, 5, 159-165. [CrossRef] [PubMed]

4. Bartels, H.; Oldigs, H.-D.; Günther, E. Use of saliva in monitoring carbamazepine medication in epileptic children. Eur. J. Pediatr. 1977, 126, 37-44. [CrossRef] [PubMed]

5. Rylance, G.W.; Butcher, G.M.; Moreland, T. Saliva carbamazepine levels in children. Br. Med. J. 1977, $2,1481$. [CrossRef] [PubMed]

6. MacKichan, J.J.; Duffner, P.K.; Cohen, M.E. Salivary concentrations and plasma protein binding of carbamazepine and carbamazepine 10,11-epoxide in epileptic patients. Br. J. Clin. Pharmacol. 1981, 12,31-37. [CrossRef]

7. Arranz Peña, M.I.; Saenz Lope, E. Can a single measurement of carbamazepine suffice for therapeutic monitoring? Clin. Chem. 1987, 33, 812-813. [CrossRef] 
8. Petit, P.; Lonjon, R.; Cociglio, M.; Sluzewska, A.; Blayac, J.P.; Hue, B.; Alric, R.; Pouget, R. Carbamazepine and its 10,11-epoxide metabolite in acute mania: Clinical and pharmacokinetic correlates. Eur. J. Clin. Pharmacol. 1991, 41, 541-546. [CrossRef]

9. Callaghan, N.; Goggin, T. A comparison of plasma and saliva levels of carbamazepine and phenytoin as monotherapy. Ir. J. Med. Sci. 1984, 153, 170-173. [CrossRef]

10. Datar, P.A. Quantitative bioanalytical and analytical method development of dibenzazepine derivative, carbamazepine: A review. J. Pharm. Anal. 2015, 5, 213-222. [CrossRef]

11. Patsalos, P.N.; Berry, D.J. Therapeutic drug monitoring of antiepileptic drugs by use of saliva. Ther. Drug Monit. 2013, 35, 4-29. [CrossRef] [PubMed]

12. Patsalos, P.N.; Spencer, E.P.; Berry, D.J. Therapeutic drug monitoring of antiepileptic drugs in epilepsy: A 2018 Update. Ther. Drug Monit. 2018, 40, 526-548. [CrossRef] [PubMed]

13. Cohen, H. Carbamazepine. In Casebook in Clinical Pharmacokinetics and Drug Dosing; Cohen, H., Ed.; McGraw-Hill: New York, NY, USA, 2015; Available online: http://accesspharmacy.mhmedical.com/content. aspx?bookid=1514\&sectionid $=88803447$ (accessed on 8 January 2020).

14. Ruiz, M.E.; Conforti, P.; Fagiolino, P.; Volonté, M.G. The use of saliva as a biological fluid in relative bioavailability studies: Comparison and correlation with plasma results. Biopharm. Drug Dispos. 2010, 31, 476-485. [CrossRef] [PubMed]

15. Dwivedi, R.; Singh, M.; Kaleekal, T.; Gupta, Y.K.; Tripathi, M. Concentration of antiepileptic drugs in persons with epilepsy: A comparative study in serum and saliva. Int. J. Neurosci. 2016, 126, 972-978. [CrossRef]

16. Rylance, G.W.; Moreland, T.A.; Butcher, G.M. Carbamazepine dose-frequency requirement in children. Arch. Dis. Child. 1979, 54, 454-458. [CrossRef]

17. Moreland, T.A.; Priestman, D.A.; Rylance, G.W. Saliva carbamazepine levels in children before and during multiple dosing. Br. J. Clin. Pharmacol. 1982, 13, 647-651. [CrossRef]

18. Hockings, N.; Pall, A.; Moody, J.; Davidson, A.V.; Davidson, D.L. The effects of age on carbamazepine pharmacokinetics and adverse effects. Br. J. Clin. Pharmacol. 1986, 22, 725-728. [CrossRef]

19. Miles, M.V.; Tennison, M.B.; Greenwood, R.S. Intraindividual variability of carbamazepine, phenobarbital, and phenytoin concentrations in saliva. Ther. Drug Monit. 1991, 13, 166-171. [CrossRef]

20. Carvalho, J.; Rosado, T.; Barroso, M.; Gallardo, E. Determination of antiepileptic drugs using dried saliva spots. J. Anal. Toxicol. 2019, 43, 61-71. [CrossRef]

21. Chee, K.Y.; Lee, D.; Byron, D.; Naidoo, D.; Bye, A. A simple collection method for saliva in children: Potential for home monitoring of carbamazepine therapy. Br. J. Clin. Pharmacol. 1993, 35, 311-313. [CrossRef]

22. Dziurkowska, E.; Wesolowski, M. Solid phase extraction purification of saliva samples for antipsychotic drug quantitation. Molecules 2018, 23, 2946. [CrossRef] [PubMed]

23. Dziurkowska, E.; Wesolowski, M. Simultaneous quantification of antipsychotic and antiepileptic drugs and their metabolites in human saliva using UHPLC-DAD. Molecules 2019, 24, 2953. [CrossRef] [PubMed]

24. Alexishvili, M.M.; Rukhadze, M.D.; Okujava, V.M. Simultaneous determination of carbamazepine and carbamazepine 10,11-epoxide by using microcolumn HPLC: Study of pharmacokinetics of carbamazepine in a volunteer. Biomed. Chromatogr. 1997, 11,36-41. [CrossRef]

25. Dordević, S.; Kilibarda, V.; Stojanović, T. Determination of carbamazepine in serum and saliva samples by high performance liquid chromatography with ultraviolet detection. Vojnosanit. Pregl. 2009, 66, 347-352.

26. Rukhadze, M.D.; Tsagareli, S.K.; Sidamonidze, N.S.; Meyer, V.R. Cloud-point extraction for the determination of the free fraction of antiepileptic drugs in blood plasma and saliva. Anal. Biochem. 2000, 287, 279-283. [CrossRef] [PubMed]

27. Goldsmith, R.F.; Ouvrier, R.A. Salivary anticonvulsant levels in children: A comparison of methods. Ther. Drug Monit. 1981, 3, 151-157. [CrossRef] [PubMed]

(C) 2020 by the authors. Licensee MDPI, Basel, Switzerland. This article is an open access article distributed under the terms and conditions of the Creative Commons Attribution (CC BY) license (http://creativecommons.org/licenses/by/4.0/). 